\title{
Targeted radiofrequency treatment - Oncothermia application in non- oncological diseases as special physiotherapy to delay the progressive development
}

Oncothermia (OTM) is based on electromagnetic interactions with the living matter. Its nano-targeting approach [establishes a new paradigm, which could be applied not only in case of malignancies but in other diseases, when the non-selective conditions [are valid. This technique is well proven from the laboratory level to the clinical applications. Oncothermia method ignites the natural processes to eliminate them from the system, re-establishing the communication harmony between the cells. This method will lead us to the treatment of some non-malignant diseases too. Our aim is to use OTM on the common basis of equilibrium demand; and use the recognition of the deviations from the complex harmony of the organism or its part for selection to act properly.

Study protocol and Method- Oncotherm was successfully applied for Lyme-disease for low-back pain, for Peyronie disease, and for Dupuytren's contracture, too. We made more extended study, proving in details the applicability of the OTM in these diseases, especially in the situations when traditional Chinese Medicine (TCM) is also applicable. Our special permanent acupuncture method well fits to the complementary applications.

Results, Discussion-The synergy of the ancient knowledge and the high-tech state-of-art of the medical knowledge could be established with this research Recognition of the distortions in the healthy tissue have some common principles and possibilities in TCM and OTM: the loss of complexity of the living organization is recognized by both the methods.. OTM application is a useful, harmless additional complementary treatment for management of selected diseases. Our objective is giving western trained physicians clinical applications of modern (Oncothermia-Booster) as a physiotherapeutic - equipment to accommodate accelerating interests in modern complex treatment of chronic low back pain and Dupuytren's contracture.

Conclusion- In recent study data verified the relevant end-points of the study: the safety, the quality of life (QoL), the shortened rest time, duration of painless state, cost/benefit ratio.

\section{Keywords: physiotherapy, oncothermia, Dupuytran contracture, aspecific low back pain}

\section{Abbreviations}

OTH: Oncothermy; LBP: low back pain; TCM: Traditional Chinese Medicine; VAS: Visual Analog Scale; QoL: Quality of Life

\section{Introduction}

Oncothermia (OTM) is based on electromagnetic interactions with the living matter [1]. Its nano-targeting approach [2,3] establishes a new paradigm, which could be applied not only in case of malignancies but also in other diseases, when the non-selective conditions [4] are valid. Oncothermia method ignites the natural processes to eliminate them from the system reestablishing the communication harmony between the cells. Our aim is to use OTM on the common basis of equilibrium demand; and use the recognition of the deviations from the complex harmony of the organism or its part for selection to act properly. This method will lead us to the treatment of some non-malignant diseases, too. This technique is well-proven from the laboratory level to the clinical applications [5].

Method and procedure
Gabriella Hegyi*, Molnar Istvan, Mate Ágnes \& Petrovics Gábor.

Pecs University, CAM Department, Doctoral School, Hungary, Pecs, Vörösmarty u.4. Hungary

*Author for correspondence: drhegyi@hu.inter.net 
We decided to apply Oncothermia in the following 2 group of non-malignant disease:

\section{- Dupuytren's contracture}

Also known as Morbus Dupuytren's, or Dupuytren's disease and slang term "Viking disease" or "Celtic hand", is a fixed flexion contracture of the hand due to a palmar fibromatosis, where the fingers bend towards the palm and cannot be fully extended (straightened). The ring finger and little finger are the fingers most commonly affected. The middle finger may be affected in advanced cases, but the index finger and the thumb are not affected as frequently. It is an inherited proliferative connective tissue disorder that involves the hand's palmar fascia. It is named after Baron Guillaume Dupuytren, the surgeon who described an operation to correct the affliction in the Lancet in 1831. Dupuytren's contracture progresses slowly and is often accompanied by some aching and itching. In patients with this condition, the palmar fascia thickens and shortens so that the tendons connected to the fingers cannot move freely. Incidence increases after age 40; at this age, men are affected more often than women. Beyond 80 the gender distribution is about even. The progressive situation can be improved by operation in stage No, N1 and other alternative therapies can be tried, like Quercetin, Bromelain, DMSO, MSM, Acetylcarnitin $\mathrm{HCl}$, PABA, Nattokinase, Vitamin E (1940) Copper, Vitamin C, Massage. The palmar fascia becomes hyperplastic and contracts by time to time.

\section{- Non-specific low back pain}

(LBP), also known as lower back pain or lumbago, is a common disorder involving the muscles and bones of the back. Low back pain may be classified by duration as acute (pain lasting less than 6 weeks), sub-chronic (6 to 12 weeks), or chronic (more than 12 weeks). The condition may be further classified by the underlying cause as mechanical, nonmechanical, or referred pain. In most episodes of low back pain, a specific underlying cause is not identified or even looked for, with the pain believed to be due to mechanical problems such as muscle or joint strain. If the pain does not go away with conservative treatment or if it is accompanied by "red flags" such as unexplained weight loss, fever, or significant problems with feeling or movement, further testing may be needed to look for a serious underlying problem.
In most cases, imaging tools such as X-ray computed tomography are not useful and carry their own risks. Some low back pain is caused by damaged intervertebral discs, and the straight leg raise test is useful to identify this cause. In those with chronic pain, the pain processing system may malfunction, causing large amounts of pain in response to non-serious events. Despite this, the use of imaging in low back pain has increased. The treatment of acute non-specific low back pain is typically with conservative measures: as the use of simple pain medications and the continuation of as much normal activity as the pain allows without bed rest. Chronic non-specific lower back pain needs more management for relieving permanent pain. The Booster, which is the unique instrument, was developed first for oncology, but can nevertheless also be successfully used in other medical fields in addition to oncology, in the follows: in fields such as rheumatology, sports medicine, neurology and neurosurgery, dermatology and analgesic therapy. We know that Oncotherm was successfully applied previously for Lymedisease, [6] for low-back pain, [7] for Peyronie disease, [8] and for Dupuytren's contracture, [9] too. The number of patients was small one in the last mentioned study; therefore we made more extended study, proving in details the applicability of the OTM in these diseases, especially in the situations when traditional Chinese Medicine (TCM) is also applicable. Our special acupuncture method [10] well fits to the complementary applications. We enlarged number of selected patients suffering from Dupuytren's contracture in different stages of disease to investigate whether progression without side-effect could be stopped with elevation of QoL of patients and non-specific low back pain lasting more than 4 weeks where we used focused radiofrequency heat therapy as special physiotherapy.

The study for selected diseases was performed according to Helsinki Declaration and local ethical approval.

Patient selection was made by rheumatologist outpatient's clinic. The period of study was 2012-2015.

We selected 76 patients suffering from Dupuytren's contracture in different stages (FIGURES 1 and 2). 

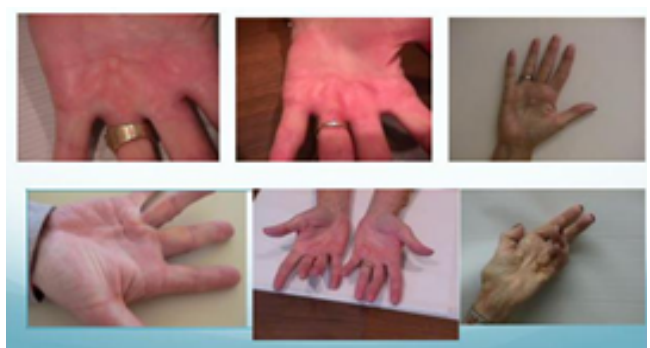

Figure 1: Dupuytren's contracture in different stages.

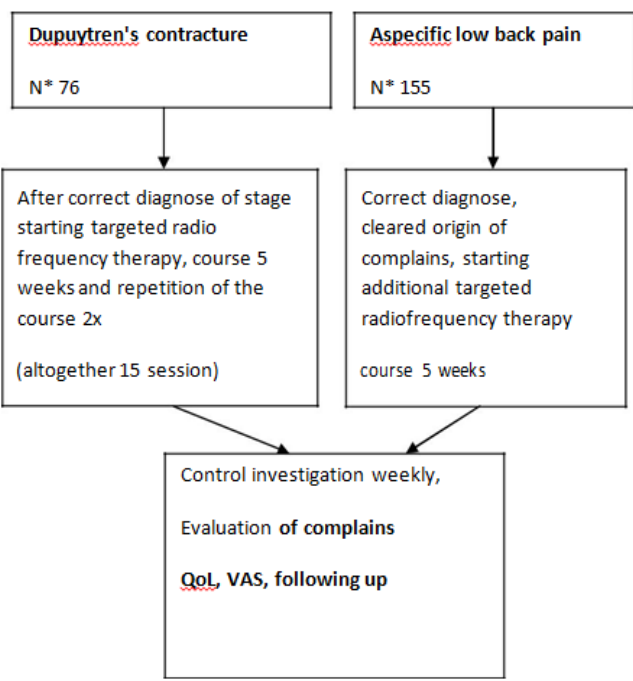

Figure 2: Flowchart for procedure.

The selected patients were suffering from N0, N1, N2 stages received 2 times/week special booster-physiotherapy with Instrument (Booster, Oncotherm GmbH, Germany), 30 W, 25 minutes, 1 course is 4 weeks ( 8 session), after $1 / 2$ years it was repeated 3 times. The primer outcome measurement was "top of plate test”, (FIGURE 3) painless extension of palm. Second outcome measurements were QoL, VAS of patients.

Also, we selected 155 patients suffering from aspecific low back pain, including verified ischias, discopathy, disc herniation and degenerative disease. Excluding criteria were ruptured disc herniation, caudal syndrome, malignancy - (bone metastasis from lung, breast, prostate, thyroid, among others), infectious (osteomyelitis; abscess) (FIGURE 4).

\section{"Table top test"}

With this test, the patient places his hand on a table.

If the hand lies completely flat on the table, the test is considered negative.

If the hand cannot be placed completely flat on thetable, but thereisaspacebetween thetableanda part of the hand as big as the diameter of a ballpoint pen, the test is considered positive

and surgery or other treatment may be indicated.

Additionally, finger joints may become fixed and rigid.

The selected patients received 2-times/ week special booster-physiotherapy (with instrument Booster Oncotherm $\mathrm{GmbH}$, Germany), $30 \mathrm{~W}, 25$ minutes, 1 course is 4 weeks ( 8 sessions), and after $1 / 2$ years it was repeated 3 times. Additionally, all patients received mesotherapy (neuraltherapy by local anaesthetic Procain $0.5 \%, 2-3 \mathrm{ml} /$ session and acupuncture (permanent monofilamentum insertion 1 time / month according to TCM protocol and protocol of Neuraltherapy) (FIGURES 5 and 6). Questionnaire on pain and QoL was filled in all sessions during physical examination. Visual Analog Scale (VAS), "Top of plate test" was registered during the all period of treatments.

\section{Results}

We recognized in N0 and N1st stage of Dupuytren's cases significant delay of progression, moderately in 2 nd, no changes in progressive stages after 2 years followup. According to delayed progression in VAS can be seen result (FIGURE 7). The

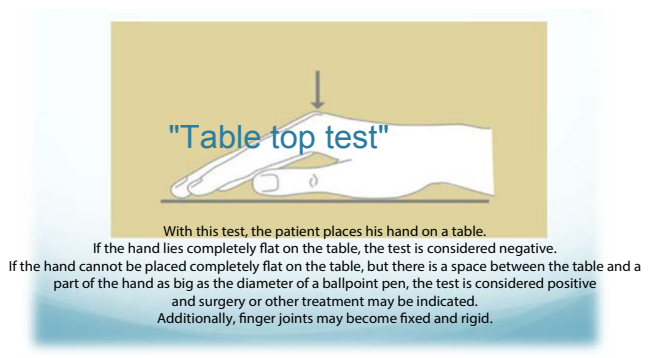

Figure 3: Table Top Test.

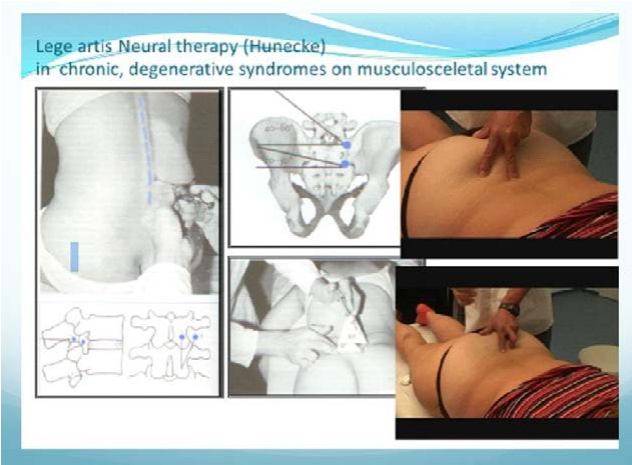

Figure 4: Aspecific lower back pain. 


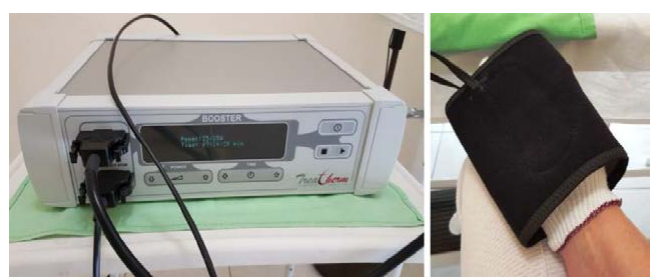

Figure 5: Course of treatment with booster instrument.

Aspecific lower back pain

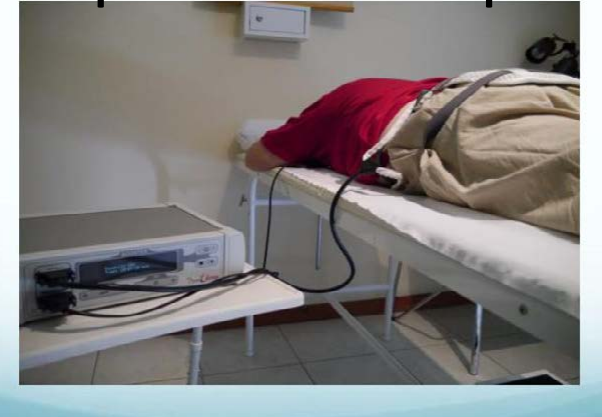

Figure 6: Low back pain treatment.

following additional therapies used to apply for Dupuytren's cases Quercetin, Bromelain, DMSO, MSM, Acetylcarnitin $\mathrm{HCl}$, PABA, Nattokinase, Vitamin E (1940) Copper, Vitamin C, usual continuous massage therapy.

\section{Discussion}

The second investigated of non-specific low back pain, better results were achieved in cases originated from ischialgy, chronic degenerative lumbar disc cases, low back pain caused by postoperative edema. The rest time out of work was less. The low back patents needed fewer intakes of analgesic pills (FIGURE 8).

According to our experience the "targeted radiofrequency-physiotherapy as Booster" application is free of side effects (but skill, practice is necessary), harmless, well-tolerable by patients, easy to apply by skilled administration. It is advised to consider that complementary treatment of selected diseases is valuable for introducing into daily rheumatologic practice. Also we recognized the fewer intakes of medicaments in cases during sessions of low back pain, which is important to avoid unnecessary side effects of medicaments (NSAID).

\section{Conclusion}

The targeted radiofrequency therapy is used as a supplement to pharmacotherapy and general drug treatments. It is not intended to be a stand-alone treatment device, but should be used in a supporting role with drug treatments to enhance their effects.

The purpose of the Booster is to increase the blood flow in the treatment area. Selection at cellular level does not occur, only a heating of the deep layers of tissue in the region where the electrode is positioned. The deep-heating effect is a result of Joule loss and leads to vascular dilatation in the treatment area, that in turn, improves blood perfusion and thus the drugs (and more oxygen) are transported to the treatment area. The temperature in this area is $37-39^{\circ} \mathrm{C}$ (moderate, classic

Hyperthermia), and this is the optimum temperature for the booster's effect. It can be accepted the improvement due to increased fibroblast activity and increased capillary growth, increased nutrient concentration and metabolic activity, synergetic increase in the field-dependent effects, (optimization of membrane stimulation and activation of signal channels etc.). Increased reactions to heat and field exposure (mainly the development of heat shock proteins, HSP), increased venous and lymphatic flow causing changes in the physical properties of the tissue finally.

Recognition of the distortions in the healthy tissue has some common principles and possibilities in TCM and OTM: the loss

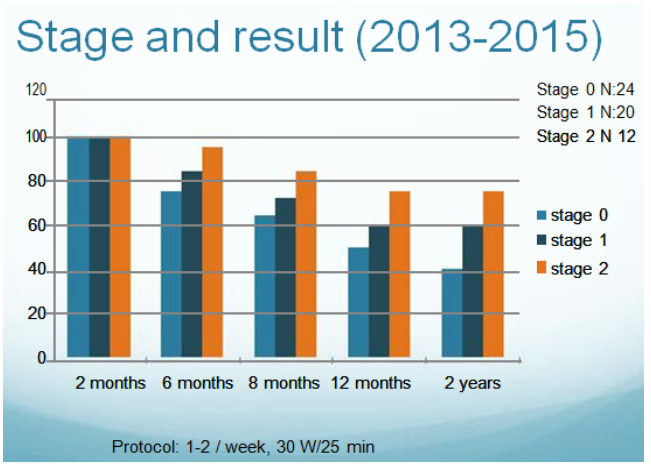

Figure 7: Results in Dupuytrans contracture.

\section{MESOTHERAPY+BOOSTER $(n=155)$ VAS $(2013-2015)$}

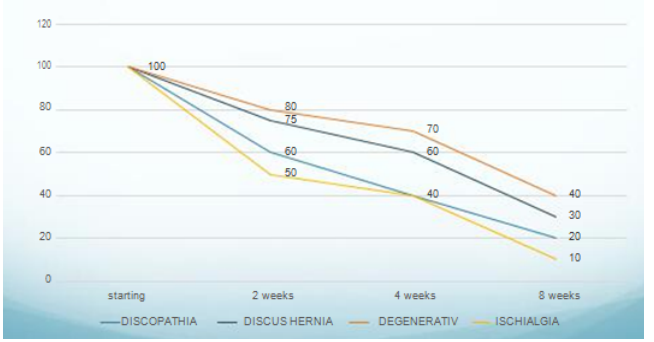

Figure 8: Results in low back pain. 
of complexity of the living organization is recognized by both methods. The synergy of the ancient knowledge and the high-tech state-of-art of the medical knowledge could be established with this research. The positive result of this study was the recognition to apply the radiofrequency heat therapy for non-malignant, developing connective sittue diseases with positive result. The limitation of this study is lack of doubleblind control and lack of "sham" application.
We need further researches in the future to clarify the exact undergoing process in selected disease group, but results show improvement and better QoL on regarded diseases, therefore it is advisable to use this physiotherapeutic method in daily practice of mentioned disease after careful selection $[11,12]$.

The present scientific contribution is dedicated to the 650th anniversary of the foundation of the University of Pécs, Hungary.

\section{REFERENCES}

Szasz A, Szasz N, Szasz O. OncothermiaPrinciples and Practices. Open J Biophy, 3(4), (2013).

Szasz A, Shimizu T, Kondo T. Electromagnetic effects in nanoscale range, chapter in book Cellular response to physical stress and therapeutic application Nova Science Publishers, Inc. (2013).

Szasz O, Szasz A. Oncothermia - Nanoheating paradigm. J Cancer Sci Ther. 6(4),117-121(2014).

Szasz A. Challenges and solutions in oncological hyperthermia. Thermal Med. 29(1),1-23(2013).

Andocs G, Szasz O, Szasz A. Oncothermia treatment of cancer: from the laboratory to clinic, Electromagn Biol Med 28(2), 148-65(2009).

Zais O, Szasz A. Lyme disease and oncothermia, Conference Papers in Medicine. Article ID 275013, Hindawi (2013).

Hegyi G, Jian L, Szasz A (2013) Low back pain-Complex approach of treatment by different CAM modalities, Conference Papers in Medicine.

Ballerini M, Baronzio GF, Capito G et al. Androtherm application for the Peyronie's disease, Conference Papers in Medicine. (2013).

Hegyi G. Usage of Booster (oncothermia) in rehabilitation of non-malignant conditions, 32nd Annual Conference of ICHS 2013, Panyu, China PR, (2013).

Hegyi G, Szigeti GP. Rehabilitation of Stroke Patients using Yamamoto New Scalp Acupuncture - A Pilot Study. J Alt Compl Med 18(10),1-7(2012).

Kanodia AK, Legedza ATR, Davis RB, Eisenberg DM, Phillips RS. Perceived benefit of Complementary and Alternative Medicine (CAM) for back pain: A national survey. J Am Board Fam Med 23(3),354-362(2010).

Hegyi G, Szasz O, Szasz A. Oncothermia: A New Paradigm and Promising Method in Cancer Therapies. Int J. 38(3-4), 161-197 (2013). 